\title{
PRODUÇÃO DE PESQUISA EM POLÍTICAS PÚBLICAS LOCAIS DE DESPORTO NO BRASIL E EM PORTUGAL: AGENDA EM FORMAÇÃO
}

\section{Resumo}

Edmilson Santos dos Santos

Doutorado

Universidade Federal do Vale do São Fracnisco edmilson.santos@ni

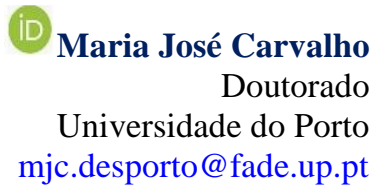

Objetivo: O presente trabalho buscou identificar se há uma agenda de pesquisa de policy analysis no âmbito dos estudos que tratam das políticas locais de desporto no Brasil e Portugal.

Método: De caráter descritivo, a pesquisa se efetivou, num primeiro momento, através de revisão sistemática que identificou 70 trabalhos sobre políticas locais de desporto nos dois países. Após o levantamento bibliográfico, elaboramos um banco de dados contemplando três critérios de classificação dos trabalhos: caracterização da agenda de pesquisa, modelo teórico e modelo metodológico.

Originalidade/Relevância: No Brasil e em Portugal, o poder público local tem sido um promotor importante de políticas públicas de desporto. No entanto, essa realidade ainda foi não explorada no sentido de identificar a existência de uma agenda de pesquisa no âmbito da policy analysis.

Resultados: Considerando que os estudos legítimos da policy analysis buscam explicar a existência das políticas e as variáveis que afetam sua formação, implementação e desempenho, os trabalhos não foram capazes ainda de constituírem uma agenda de pesquisa. A utilização de metodologias descritivas na totalidade das análises evidencia que os estudos compõem ainda a primeira geração de trabalhos.

Contribuições teóricas/metodológicas: O estudo indica a necessidade de os trabalhos que analisam as políticas locais de desporto nos dois países superarem o aspecto descritivo ( $1^{\text {a }}$ geração) e passarem a identificar as variáveis que afetam seus resultados.

Palavras-chave: Desporto. Políticas Públicas. Agenda de Pesquisa. Governo Local.

\section{Cite como}

American Psychological Association (APA)

Santos, E. S., \& Carvalho, M. J. (2020). Produção de pesquisa em políticas públicas locais de desporto no Brasil e em Portugal: agenda em formação. PODIUM Sport, Leisure and Tourism Review, São Paulo, 9(2), 1-14. https://doi.org/10.5585/podium.v9i2.16567. 


\title{
RESEARCH PRODUCTION ON LOCAL PUBLIC SPORT POLICIES IN BRAZIL AND PORTUGAL: AGENDA IN DEVELOPMENT
}

\begin{abstract}
Objective: This study aimed to identify if there is a policy analysis research agenda within the studies dealing with local sports policies in Brazil and Portugal.

Method: This descriptive research was carried out initially through a systematic review that identified 70 studies on local sports policies in both countries. After the bibliographical survey,we elaborated a database contemplating three classification criteria: characterization of the research agenda, theoretical model and methodological model.
\end{abstract}

Originality/Relevance: In Brazil and Portugal, the local public power has been an importante sponsor of public sport policies. However, this reality has not yet been explored in order to identify the existence of a research agenda within policy analysis.

Results: Considering that the legitimate studies of policy analysis seek to explain the existence of policies and the variables that affect their formation, and performance, the study has not yet been able to constitute a research agenda. The use of descriptive methodologies in all analyses shows that the studies still represent the first generation of researches.

Theoretical/methodological contributions: The study indicates the need for the papers that analyze local sports policies in both countries to overcome the descriptive aspect (first generation) and begin to identify which variables affect their results.

Keywords: Sport. Policy. Research Agenda. Local Government.

\section{PRODUCCIÓN DE INVESTIGACIÓN EN POLÍTICAS PÚBLICAS DEPORTIVAS LOCALES EN BRASIL Y PORTUGAL: AGENDA DE FORMACIÓN}

\section{Resumen}

Objetivo: El presente estudio buscó identificar si existe una agenda de investigación de análisis de políticas dentro del alcance de los estudios que abordan las políticas deportivas locales en Brasil y Portugal.

Método: esta investigación descriptiva se realizó inicialmente a través de una revisión sistemática que identificó 70 estudios sobre políticas deportivas locales en ambos países. Después de la encuesta bibliográfica, elaboramos una base de datos que contempla tres criterios de clasificación: caracterización de la agenda de investigación, modelo teórico y modelo metodológico.

Originalidad / Relevancia: En Brasil y Portugal, el poder público local ha sido un patrocinador importante de las políticas deportivas públicas. Sin embargo, esta realidad aún no se ha explorado para identificar la existencia de una agenda de investigación dentro del análisis de políticas.

Resultados: Considerando que los estudios legítimos de análisis de políticas buscan explicar la existencia de políticas y las variables que afectan su formación y desempeño, el estudio aún no ha sido capaz de constituir una agenda de investigación. El uso de metodologías descriptivas en todos los análisis muestra que los estudios todavía representan la primera generación de investigaciones. 
Contribuciones teóricas / metodológicas: el estudio indica la necesidad de que los documentos que analizan las políticas deportivas locales en ambos países superen el aspecto descriptivo (primera generación) y comiencen a identificar qué variables afectan sus resultados.

Palabras clave: deporte. Política. Agenda de investigación. Gobierno local.

\section{Introdução}

Brasil e Portugal, apesar das diferenças culturais, históricas e institucionais, passaram por um longo período de cerceamento das liberdades democráticas e, mesmo com as turbulências institucionais nesse período, estão buscando consolidar suas democracias e garantir a efetividade dos direitos. Os dois países produziram mudanças institucionais importantes no terreno esportivo: a constitucionalização do direito ao desporto no período de redemocratização nos anos 70/80 e a esfera local de poder como a mais adequada para atender às necessidades dos cidadãos. De forma adicional, são países que têm realizado intercâmbio acadêmico no âmbito das análises da gestão pública e privada do desporto.

As prefeituras, no Brasil, e as câmaras municipais ${ }^{1}$, em Portugal, têm assumido papel estratégico na formulação e implementação de políticas de desporto, aspecto esse que não tem passado desapercebido pela comunidade de especialistas que analisam as políticas públicas de desporto nos dois países. Apesar das diferenças em termos de forma de Estado (Brasil, Estado Federalista, e Portugal, Estado Unitário), nos dois casos o poder local tem assumido papel importante na oferta de políticas públicas de esporte.

Há diversidades e especificidades a serem consideradas quando incorporamos a esfera local como espaço de investigação sobre as políticas públicas de desporto. Os condicionantes fiscais (capacidade de arrecadação e de gasto), a gestão política e o comportamento dos stakeholders $^{2}$ produzem um cenário diverso à implementação da política. São 5.570 prefeituras no Brasil e 308 câmaras municipais em Portugal a experimentarem diferentes comportamentos políticos em relação à matéria, o que cria um ambiente de investigação muito rico em termos de políticas públicas desportivas.

\footnotetext{
${ }^{1}$ As câmaras municipais em Portugal têm função diferente das brasileiras, que são exclusivamente responsáveis pela produção legislativa. Em Portugal, elas também assumem funções executivas, de gestão das políticas.

${ }^{2}$ Donaldson \& Preston (1995, p.85) definem os stakeholders como "[...] pessoas ou grupos com interesses legítimos nos processos e aspectos substantivos de uma atividade corporativa". De forma simplificada, são os interessados pelas políticas que buscam interferir nos processos.
} 
Do ponto de vista da policy analysis ${ }^{3}$, conhecemos muito pouco as características temáticas, teóricas e metodológicas assumidas pelos estudos. Não sabemos se são apenas proliferação de estudos de caso, como destaca Melo (1999), ou se já é possível observar uma agenda de pesquisa consolidada que busca compreender o resultado da política a partir das variáveis intervenientes neste processo. Como resume Howlett, Ramesh e Perl, (2009), o estudo examina as causas, os pressupostos e os processos que permitiram a implementação de uma determinada política (ou sua inação). Ou seja, busca compreender quais fatores afetam as políticas e como afetam (Goodin, Rein, \& Moran, 2009).

Ao tecer observações sobre o crescimento do volume das publicações, Souza (2003) faz uma ressalva importante e aponta que a acumulação horizontal de conhecimento não leva necessariamente à produção de uma agenda de pesquisa. Isso porque, como destaca Arretche (2003), uma agenda de pesquisa deve permitir identificar quais são as questões legítimas e os procedimentos teóricos e metodológicos que demarcam o campo de investigação.

Analisando os trabalhos de políticas públicas no Brasil, Frey (2000) identifica que a leveza teórica e metodológica dos estudos, de forte viés descritivo, pode produzir apenas estudos empíricos preliminares, não chegando a constituir uma agenda de pesquisa. Na mesma direção, Arretche (2003) aponta que uma coleção de fatos não é suficiente para a constituição da agenda de pesquisa. São pesquisas que, geralmente, estão mais preocupadas em conhecer a forma como são desenvolvidas as políticas e seu sucesso ou fracasso. Para Souza (2003), essas análises descritivas representam a primeira geração de trabalhos, enquanto a segunda já é capaz de identificar as variáveis que impactam o resultado da política pública.

Passados mais de 30 anos da promulgação das constituições, de intensa atividade política no nível local no Brasil e em Portugal em matéria desportiva e de estudos que buscam conhecer esse fenômeno, torna-se importante conhecer o estágio de desenvolvimento da agenda de pesquisa. Considerando a experiência acumulada nos dois países no âmbito da produção do conhecimento em políticas públicas locais de desporto, o presente trabalho buscou identificar se há uma agenda de pesquisa de policy analysis no âmbito dos estudos que tratam das políticas locais de desporto no Brasil e Portugal. Para tanto, observamos três aspectos importantes: a análise sobre as questões de pesquisa, as crenças teóricas e as metodológicas.

\footnotetext{
${ }^{3}$ A especificidade da policy analysis está em analisar a inter-relação entre as instituições políticas, o processo político e os conteúdos de política (Frey, 2000). Diferentemente dos estudos descritivos, a policy analysis busca evidências sobre o impacto efetivo da variável independente na variável dependente. Ou seja, encontrar uma explicação à realização da política pública.
} 
Para desenvolvermos o trabalho, foram organizadas quatro etapas acrescidas da introdução. Num primeiro momento, apreciamos as análises que têm sido realizadas no Brasil e em Portugal sobre a produção acadêmica no âmbito das políticas públicas de esporte. Logo após, apresentamos o debate atual em torno da agenda de pesquisa em policy analysis. Após a descrição metodológica do estudo, a discussão sobre os achados da investigação e, por último, as conclusões.

\section{Referencial teórico}

As análises sobre as políticas públicas

Há muito que a preocupação em conhecer como tem se constituído o campo de investigação no âmbito das políticas públicas de desporto lato sensu vem sendo perseguida por pesquisadores de políticas públicas de desporto, especialmente no Brasil. Em linhas gerais, os estudos tentam caracterizar a produção na área por meio de diferentes aspectos. Excetuando Rijo (2018), que analisou aspectos associados aos personagens e às instituições, não há trabalhos em Portugal que façam análise com esse escopo.

No Brasil pós-constituinte (Constituição Federal de 1988), o primeiro trabalho nessa linha apontou em suas conclusões que esse campo de investigação estava alcançando legitimidade científica (Santos, Batista \& Araújo, 2007). Os autores reconheceram a existência de uma agenda de pesquisa. Starepravo, Afonso \& Ferreira (2008) também reconhecem a legitimidade científica do campo, mas alertam como um dos pontos mais frágeis das discussões a não utilização de referências consistentes. No cenário internacional, Houlihan (2005) também já havia alertado sobre essa dificuldade das análises de políticas esportivas.

Ao identificar que a produção no âmbito das políticas públicas desportivas havia crescido, Amaral \& Pereira (2009) questionam se esses trabalhos já podem ser considerados pesquisas científicas. Reconhecendo a evolução das análises, os autores alertam para a necessidade de superarmos seu aspecto descritivo: "há uma recorrência em pesquisar sobre estruturas governamentais, programas de ação sem uma devida atenção àquilo que há de recorrente nessas políticas e os enlaces possíveis com outras dimensões da política” (p. 52).

Apesar de não utilizar um modelo apriorístico, Starepravo, Nunes \& Marchi Jr. (2009) buscam descrever a agenda de pesquisa (temáticas legítimas e perspectivas teóricas) a partir 
Santos, E. S., \& Carvalho, M. J. (2020). Produção de pesquisa em políticas públicas locais de desporto no Brasil e em Portugal: agenda em formação

daquilo que os trabalhos apresentaram. Duas questões foram destacadas: a existência de trabalhos que não apresentam diálogo teórico consistente e, entre os que apresentam maior consistência, uma postura crítica ao neoliberalismo. Ao finalizarem, os autores sentenciam: "para que haja um avanço significativo nas produções, necessitamos de referenciais consistentes de análises" (p. 10). Ou seja, reconhecem a fragilidade teórica dos estudos quando comparado, especialmente, com a produção nos ramos da ciência com maior tradição em análise de políticas.

Ao analisarem a produção em políticas públicas de desporto e lazer no Brasil, Amaral, Ribeiro \& Silva (2014) confirmam amadurecimento do campo com a maior aproximação de outros campos disciplinares. Apesar de fazer uma análise mais restritiva, apenas uma revista científica, Sousa et al. (2016) apontaram a baixa priorização quanto aos aspectos teóricos e metodológicos nos trabalhos.

Como podemos observar, os trabalhos destacados aqui reconhecem a existência de um campo específico de análise, as políticas públicas de desporto e de lazer, mas, em linhas gerais, assumem que existe uma lacuna associada às questões teóricas e metodológicas. A agenda é reconhecida por parâmetros diferentes daqueles aceitos pela policy analysis que exigem que o corpo teórico e as estratégias metodológicas estabeleçam de forma clara a relação entre a variável dependente e as variáveis independentes.

Em trabalho realizado em 2003, Souza já reconhecia o avanço das pesquisas em políticas públicas no Brasil, mas evidenciou o "uso excessivo de narrativas pouco pautadas por modelos ou tipologias de políticas públicas, por teorias próximas do objeto de análise e que mantêm uma leveza metodológica exagerada" (p. 17). Realidade muito próxima daquilo que foi destacado pelos analistas de políticas do desporto aqui apresentados.

Ou seja, os trabalhos identificados como sendo de policy analysis precisam ser acompanhados de um aporte teórico que, além de explicar as variáveis que afetam a política, deve vir junto de um desenho metodológico que permita fazer inferências seguras sobre esse processo. Sendo assim, a temática de estudo deve ser capaz de garantir o adequado funcionamento das premissas teórica e metodológica (Howlett, Ramesh \& Perl, 2013).

\section{Temáticas e modelos teóricos e metodológicos}

O desenvolvimento do campo de análise das políticas públicas de desporto (e podemos especificar aqui aqueles desenvolvidos pelos governos locais) já permite hoje desenharmos um 
modelo analítico da agenda de pesquisa que identifique as possibilidades de temáticas de estudo e os modelos teóricos e metodológicos usuais.

A identificação da temática ajuda a identificar as questões legítimas de uma determinada comunidade científica. O predicado local (das políticas públicas locais de desporto) não produz uma grande distinção em relação a outros ambientes de produção da política, como o estadual, o regional e o nacional. Em todos esses casos, são governos pressionados por recursos escassos, enfrentando problemas públicos no campo esportivo.

No entanto, Frey (2000) identificou algumas limitações em estudos que buscam abordar a experiência local brasileira. $\mathrm{O}$ primeiro se refere às limitações do conhecimento científico quanto aos arranjos institucionais e as características dos processos políticos municipais. No âmbito do desporto, não temos conhecimento dos diferentes arranjos institucionais (formas de gestão da política e da diversidade normativa) e os impactos desses arranjos no processo político. Segundo, o crescimento contínuo e desordenado dos atores societais locais (movimentos sociais, associações de moradores e stakeholders esportivos) pode ter forte impacto nas arenas municipais. Acrescida a essas dificuldades, está outra de ordem mais geral, que é a falta de consolidação político-ideológica dos diferentes atores: população, eleitores, políticos e partidos.

Em Portugal, há um conjunto de regras que estabelecem maior precisão sobre os limites e possibilidades da esfera local (câmaras municipais) na implementação das políticas. Principalmente pela existência de uma lei de diretrizes e bases do desporto ${ }^{4}$ e da Lei das Autarquias Locais ${ }^{5}$ que melhor estruturam o comportamento (responsabilidades) dos decisores quanto à implementação de políticas desportivas pela esfera local.

Para caracterização das temáticas de estudo (agenda de pesquisa), construímos, com base na experiência dos autores, um quadro modelo (Quadro 1) que contempla sete dimensões da política pública: (1) Institucional; (2) Geográfica; (3) Dimensão da política; (4) Fase da política; (5) Modelo de descentralização; (6) Manifestação desportiva; (7) Ambiente legislativo.

A dimensão Institucional aponta o responsável do Estado pela ação: executivo, legislativo ou judiciário. A Geográfica contempla duas realidades: os estudos de caso, focando apenas uma cidade, e os estudos comparativos. Os estudos comparativos se dividem em quatro

\footnotetext{
${ }^{4}$ Denominada Lei de Bases da Actividade Física e do Desporto, Lei n. ${ }^{\circ}$ 5/2007, de 16 de janeiro.

${ }^{5}$ Denominada Regime Jurídico das Autarquias Locais, Lei n. ${ }^{\circ} 75 / 2013$, de 12 de setembro, na redação da sua 8. ${ }^{a}$ versão, a mais atual pela Lei n. ${ }^{\circ}$ 50/2018, de 16 de agosto. Ver, entre outros, o artigo $23 .^{\circ}$, onde consta os tempos livres e o desporto como uma delas [n. ${ }^{\circ} 2$, al. f)]
} 
Santos, E. S., \& Carvalho, M. J. (2020). Produção de pesquisa em políticas públicas locais de desporto no Brasil e em Portugal: agenda em formação

realidades: um menor conjunto agregado de cidades, podendo ser Região Metropolitana ou outro conjunto de cidade (RMou); a escala estadual (BR) ou distrital (PT); a escala regional; a escala nacional. A dimensão Política busca aferir se a atenção é para uma política específica (ação, programa ou lei) ou se trata de ações genéricas de governo. A Fase da política, para efeito deste trabalho, foi considerada em três etapas: agenda/formulação, implementação e avaliação. O Modelo de descentralização busca distinguir entre as descentralizações bottom-up, políticas cuja agenda é da esfera local, e descentralizações top-down, que são políticas de outros níveis de governo implementadas localmente. A Manifestação desportiva considera o desporto rendimento (elite) e o desporto participação (para todos). Para contemplar aqueles trabalhos que tratam da política geral, definimos como sendo política desportiva. Por último, nos trabalhos que envolvem o Ambiente legislativo, observamos três questões: a produção das normas, o trabalho nas comissões e o papel dos atores na formulação e apoio às proposituras.

Quadro 1 - Caracterização dos trabalhos de análises de políticas públicas

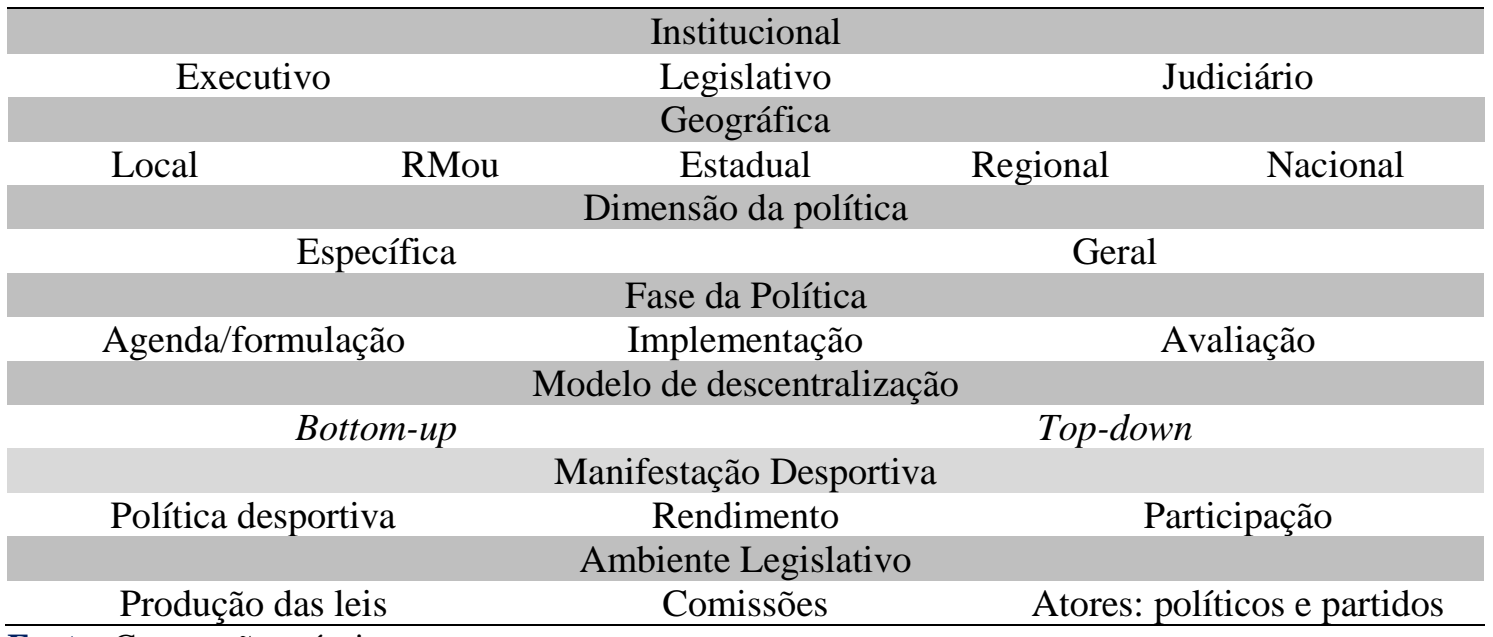

Fonte: Construção própria.

Apesar de não ser o único, o ramo da ciência que tem apresentado um conjunto de modelos teóricos à policy analysis é a ciência política. Seus modelos ajudam a termos uma compreensão mais sofisticada do processo político que permite o surgimento, a implementação e a avaliação das políticas (Houlihan, 2005; Mendes, 2013; Almeida \& Mezzadri, 2017).

Nesse sentido, utilizamos alguns dos modelos já consolidados para melhor compreender o estágio de desenvolvimento teórico dos trabalhos, conforme breve descrição apresentada no quadro 2. Para montagem do quadro, utilizamos de forma preferencial aqueles modelos teóricos 
Santos, E. S., \& Carvalho, M. J. (2020). Produção de pesquisa em políticas públicas locais de desporto no Brasil e em Portugal: agenda em formação

destacados por Howlett, Ramesh e Perl (2009). Outras referências para a elaboração foram Souza (2006), Capella (2006) e Secchi (2011).

Quadro 2 - Modelos de análise de políticas públicas do desporto

\begin{tabular}{|c|c|c|}
\hline Modelo & Análise de políticas públicas & Estudos de referência \\
\hline Incrementalismo & $\begin{array}{l}\text { O modelo é utilizado para analisar a } \\
\text { mudança na política. Seu pressuposto } \\
\text { básico é que as políticas não partem do } \\
\text { zero. Decisões marginais vão ajustando } \\
\text { o modelo de forma a garantir mudanças } \\
\text { incrementais }\end{array}$ & $\begin{array}{l}\text { Zhang, P. \& Wang, J. (2015). } \\
\text { Evolution of School Sports } \\
\text { Policy Since 1949: A Study } \\
\text { Based on Policy Texts. China } \\
\text { Sport Science, (2), 2. }\end{array}$ \\
\hline $\begin{array}{l}\text { Ciclo da Política } \\
\text { Pública }\end{array}$ & $\begin{array}{l}\text { Analisa a política como resultado de } \\
\text { uma sequência de estágios de } \\
\text { desenvolvimento da política. }\end{array}$ & $\begin{array}{l}\text { McCloy, C. (2006). The role } \\
\text { and impact of Canadian } \\
\text { federal sport hosting policies in } \\
\text { securing amateur sport } \\
\text { legacies: Case studies of the } \\
\text { past four decades. (Doctoral } \\
\text { Dissertation). University of } \\
\text { Toronto, Toronto, Canadá. }\end{array}$ \\
\hline Múltiplos Fluxos & $\begin{array}{l}\text { A política é resultado da confluência } \\
\text { dos } 3 \text { fluxos: problemas, soluções e } \\
\text { político. Quando os } 3 \text { fluxos se unem, a } \\
\text { mudança na agenda acontece. }\end{array}$ & $\begin{array}{l}\text { Piggin, J., \& Hart, L. (2017). } \\
\text { Physical activity advocacy in } \\
\text { the UK: a multiple streams } \\
\text { analysis of a hybrid policy } \\
\text { issue. Leisure Studies, 36(5), } \\
\text { 708-720. }\end{array}$ \\
\hline $\begin{array}{l}\text { Coalizão de } \\
\text { Defesa }\end{array}$ & $\begin{array}{l}\text { Cada política é composta por um } \\
\text { conjunto de subsistemas. As coalizões } \\
\text { que compõem o subsistema lutam para } \\
\text { manter a hegemonia na política e se } \\
\text { distinguem pelas crenças, ideias e } \\
\text { recursos disponíveis. }\end{array}$ & $\begin{array}{l}\text { Bueno, L. (2008). Políticas } \\
\text { Públicas do Esporte no Brasil: } \\
\text { razões para o alto rendimento. } \\
\text { (Tese Doutorado). Escola de } \\
\text { Administracãa de Empresas de } \\
\text { São Paulo, São Paulo, Brasil. }\end{array}$ \\
\hline $\begin{array}{l}\text { Equilíbrio } \\
\text { Interrompido }\end{array}$ & $\begin{array}{l}\text { Períodos longos de estabilidade são } \\
\text { interrompidos por períodos de } \\
\text { instabilidade que permitem mudar a } \\
\text { política. Mudanças que podem ser } \\
\text { incremental ou mudar profundamente a } \\
\text { política. }\end{array}$ & $\begin{array}{l}\text { Chapman, P. (2014). Policy } \\
\text { stability in a time of turbulence: } \\
\text { the case of elite sport policy in } \\
\text { England/the UK (Doctoral } \\
\text { Dissertation). Loughborough } \\
\text { University, Loughborough, } \\
\text { Reino Unido. }\end{array}$ \\
\hline $\begin{array}{l}\text { Novo } \\
\text { Gerencialismo } \\
\text { Público }\end{array}$ & $\begin{array}{l}\text { A política é resultado de um processo } \\
\text { de eficiência, de eficácia e de } \\
\text { efetividade que permite compreender a } \\
\text { política e ajustar seus resultados e tem } \\
\text { como modelo ideal instituições livres } \\
\text { de interferência política. }\end{array}$ & $\begin{array}{l}\text { McCree, R. (2009). Sport } \\
\text { Policy and the new public } \\
\text { management in the Caribbean: } \\
\text { Convergence or resurgence? } \\
\text { Public Management Review, } \\
\text { 11(4), 461-476. }\end{array}$ \\
\hline Arenas Sociais & $\begin{array}{l}\text { Empreendedores de políticas estão } \\
\text { conectados em redes que ligam os } \\
\text { indivíduos às instituições de forma a }\end{array}$ & $\begin{array}{l}\text { Moreira, N., Nascimento, D., } \\
\text { Horovitz, G., Martins, J., \& } \\
\text { Pinto, M. (2018). Quando ser } \\
\text { raro se torna um valor: o } \\
\text { ativismo político por direitos }\end{array}$ \\
\hline
\end{tabular}




\begin{tabular}{|c|c|c|}
\hline & $\begin{array}{l}\text { promover mudanças nas agendas } \\
\text { governamentais. }\end{array}$ & $\begin{array}{l}\text { das pessoas com doenças raras } \\
\text { no Sistema Único de } \\
\text { Saúde. Cadernos de saúde } \\
\text { publica, } 34 \text {. }\end{array}$ \\
\hline $\begin{array}{l}\text { Neo- } \\
\text { institucionalismo }\end{array}$ & $\begin{array}{l}\text { Enfatiza a importância das } \\
\text { instituições/regras à decisão, } \\
\text { formulação e implementação de } \\
\text { políticas públicas afetando a estratégia } \\
\text { dos atores e/ou o conteúdo das decisões } \\
\text { políticas. }\end{array}$ & $\begin{array}{l}\text { Parrish, R. (2003). The politics } \\
\text { of sports regulation in the } \\
\text { European Union. Journal of } \\
\text { European Public Policy, 10(2), } \\
246-262 \text {. }\end{array}$ \\
\hline Marxismo & $\begin{array}{l}\text { As políticas públicas refletem a luta de } \\
\text { classe da sociedade capitalista e o } \\
\text { Estado é um instrumento para manter a } \\
\text { acumulação capitalista. }\end{array}$ & $\begin{array}{l}\text { Carneiro, S. (2018). } O \\
\text { financiamento do esporte no } \\
\text { Brasil: aspectos da atuação } \\
\text { estatal nos governos Lula e } \\
\text { Dilma. Universidade de } \\
\text { Brasília, Brasília, Brasil. }\end{array}$ \\
\hline Pluralismo & $\begin{array}{l}\text { A política pública é resultado da } \\
\text { competição e colaboração entre os } \\
\text { grupos de interesse. }\end{array}$ & $\begin{array}{l}\text { Bergsgard, A. \& Norberg, R. } \\
(2010) \text {. Sports policy and } \\
\text { politics: the Scandinavian way. } \\
\text { Sport in Society, 13(4), 567- } \\
582 .\end{array}$ \\
\hline $\begin{array}{l}\text { Ciclo Político } \\
\text { Eleitoral }\end{array}$ & $\begin{array}{l}\text { Autores atuam como maximizadores de } \\
\text { utilidade e satisfação e buscam } \\
\text { conquistar aquilo que é mais vantajoso. } \\
\text { O comportamento do eleitor e do } \\
\text { político pressionam por mais } \\
\text { intervenção do Estado, formando o } \\
\text { ciclo político eleitoral. }\end{array}$ & $\begin{array}{l}\text { Santos, G. (2019) Papel dos } \\
\text { fatores políticos na decisão dos } \\
\text { gastos com esporte e lazer nos } \\
\text { municípios entre } 2002 \text { e 2016: } \\
\text { evidências para as regiões Sul } \\
\text { e Sudeste do Brasil. (Trabalho } \\
\text { de Conclusão de Curso } \\
\text { Economia), Universidade } \\
\text { Federal da Paraíba, João } \\
\text { Pessoa, Brasil. }\end{array}$ \\
\hline Top-down & $\begin{array}{l}\text { A implementação acontece de cima } \\
\text { para baixo e quem implementa apenas } \\
\text { segue protocolos já formulados não } \\
\text { cabendo qualquer alteração na política. } \\
\text { A implementação é um problema } \\
\text { organizacional. }\end{array}$ & $\begin{array}{l}\text { Santos, S., Starepravo, A., de } \\
\text { Menezes, G., \& de Melo, R. } \\
\text { (2019). Municípios e propostas } \\
\text { vulneráveis: uma análise do } \\
\text { edital 2013 do Programa } \\
\text { Esporte e Lazer da Cidade } \\
\text { (PELC). Pensar a Prática, } 22 .\end{array}$ \\
\hline Bottom-up & $\begin{array}{l}\text { Considera a complexidade da } \\
\text { implementação e privilegia a relação } \\
\text { implementador (burocrata do nível da } \\
\text { rua), usuário e interesses responsáveis } \\
\text { por reconstruir a política. A } \\
\text { implementação é um problema de } \\
\text { conflito de interesse que se expressa nas } \\
\text { opções de cada gestão local da política. }\end{array}$ & $\begin{array}{l}\text { Santos, S. (2019). A } \\
\text { descentralização em curso das } \\
\text { políticas públicas de esporte e } \\
\text { de lazer no estado do Piauí. } \\
\text { Pensar a Prática, 22. }\end{array}$ \\
\hline $\begin{array}{l}\text { Learning } \\
\text { processes }\end{array}$ & $\begin{array}{l}\text { A avaliação é percebida como ambiente } \\
\text { de aprendizagem em que todos os } \\
\text { participantes possam construir } \\
\text { mecanismos de diálogo e de construção } \\
\text { coletiva de alternativas à melhor } \\
\text { implementação. }\end{array}$ & $\begin{array}{l}\text { Mizrahi, S., Bar-Eli, M. \& } \\
\text { Galily, Y. (2008). Sport policy } \\
\text { in a transformed socio-political } \\
\text { setting: The case of Israel. } \\
\text { Identities: Global Studies in }\end{array}$ \\
\hline
\end{tabular}


Santos, E. S., \& Carvalho, M. J. (2020). Produção de pesquisa em políticas públicas locais de desporto no Brasil e em Portugal: agenda em formação

\begin{tabular}{|l|l|l|}
\hline & & $\begin{array}{l}\text { Culture and Power, 15(1), 123- } \\
145 .\end{array}$ \\
\hline $\begin{array}{l}\text { Avaliação de } \\
\text { Impacto }\end{array}$ & $\begin{array}{l}\text { Avalia os efeitos da política, seus } \\
\text { impactos, sobre a sociedade. Busca } \\
\text { avaliar a relação causal entre a política } \\
\text { e a mudança que ela anuncia. }\end{array}$ & $\begin{array}{l}\text { Burnett, C. (2001). Social } \\
\text { impact assessment and sport } \\
\text { development: social spin-offs } \\
\text { of the Australia-South Africa } \\
\text { junior sport programme. } \\
\text { International Review for the } \\
\text { Sociology of Sport, 36(1), 41- } \\
57 .\end{array}$ \\
\hline $\begin{array}{l}\text { Avaliação de } \\
\text { resultado }\end{array}$ & $\begin{array}{l}\text { Mensura os efeitos da política sobre a } \\
\text { população-alvo, se interferiu nantos, D. (2002). Uma } \\
\text { situação-problema. }\end{array}$ & $\begin{array}{l}\text { Sontribuição à discussão sobre } \\
\text { a avaliação de desempenho das } \\
\text { instituiçães federais de ensino } \\
\text { superior: uma abordagem da } \\
\text { gestão econômica. Revista } \\
\text { Contabilidade \& Finanças, } \\
13(28), 86-99 .\end{array}$ \\
\hline
\end{tabular}

Fonte: Howlett, Ramesh e Perl (2009), Souza (2006), Capella (2006) e Secchi (2011). * Trabalho não associado ao desporto.

A preocupação metodológica não é com o modelo de tratamento dos dados que pode variar em função do tipo de informação disponível e das variáveis dependentes e independentes em questão. Para a análise aqui proposta, a questão central está em se o modelo metodológico permite elaborar evidências robustas sobre o impacto da(s) variável(eis) independente(s) no resultado da política.

Os estudos descritivos, apesar de sua validade e importância, não permitem identificar as variáveis que afetam o resultado da política, como já dissertaram alguns autores destacados anteriormente no texto. Já os estudos analíticos buscam correlacionar variáveis ou estabelecer relação de causa e efeito de forma a compreendermos o impacto da variável independente na dependente (Arretche, 2003). Portanto, na avaliação metodológica dos trabalhos, apenas buscamos conhecer como se comportam em relação a esses aspectos.

\section{Metodologia}

Para análise da agenda de pesquisa em políticas públicas locais de desporto no Brasil e em Portugal, buscamos, num primeiro momento, identificar os trabalhos associados a essa temática. Sendo assim, procedemos a revisão sistemática definindo a base de dados, os critérios de inclusão e exclusão dos artigos, a verificação da acurácia e determinação da qualidade dos estudos (Sampaio \& Mancini, 2007). 
Utilizamos, para localização dos trabalhos, o Portal de Periódicos Capes, que disponibiliza diferentes bases de dados com textos publicados pelos principais editores do mundo, distribuídos em todas as áreas do conhecimento (CAPES, 2004). Para análise latu sensos das políticas desportivas, essa mesma estratégia foi utilizada por Kocian \& Silva (2016) e Rojo, Mezzadri \& Silva (2019).

A ferramenta Google Acadêmico foi utilizada de forma a corrigir possíveis lacunas dos bancos de dados bibliográficos. Giustini (2005) e Shultz (2007) avaliam que a rapidez e a amplitude das informações são pontos positivos à utilização dessa ferramenta de busca.

Fizemos quatro combinações de termos com o operador lógico AND: (1) "esporte"/ “desporto", "município", "políticas públicas"; (2) “esporte”/ “desporto”, "governo local”, "políticas públicas". (3) “esporte”/ “desporto", "poder local”, "políticas públicas”; (4) "esporte"/ "desporto", "município". A utilização alternada da palavra "esporte" e "desporto" foi para contemplar a cultura escrita dos dois países. As seguintes palavras-chave em inglês foram utilizadas, na mesma composição: "sport", "local government", "local power" e "policy".

A triagem seguiu o seguinte percurso: (a) busca dos termos no ambiente resumo e/ou assunto; (b) com palavras exatas; (c) artigos de revistas; (d) publicação nos últimos 10 anos.

Descartamos os trabalhos (critério de exclusão) que: (a) retratassem a política desportiva educacional $^{6}$; (b) referissem-se às experiências de outros países que não Brasil e Portugal; (c) tratam do perfil do gestor público; e (d) realizem diagnóstico de equipamentos desportivos fora da avaliação do papel da gestão na configuração dessa realidade.

A verificação da acurácia dos trabalhos exigiu leitura do resumo dos trabalhos, objetivando identificar a ação do governo que foi analisada. Para os resumos que não permitiam identificar os critérios de inclusão, estabelecemos o seguinte procedimento: (1) leitura da metodologia do trabalho; (2) leitura da íntegra do trabalho. Ao final foram selecionados 238 trabalhos de 2009 a 2019. A captura dos artigos foi encerrada em 30 em dezembro de 2019. Após a aferição da acurácia, restaram 70 trabalhos, sendo $62(88,57 \%)$ retratando a realidade brasileira e oito (11,43\%), a portuguesa. Os trabalhos envolveram 126 autores, 116 do Brasil e 10 de Portugal.

\footnotetext{
${ }^{6}$ Tanto no Brasil como em Portugal, as políticas de desporto educacional são fortemente impactadas pelas políticas educacionais, visto que o setor responsável pelas decisões políticas são os educacionais. No caso em pauta, nós estamos preocupados em conhecer aquelas produções associadas exclusivamente às políticas esportivas.
} 
Após o levantamento bibliográfico, elaboramos um banco de dados na planilha eletrônica Microsoft Excel (2010) contemplando a classificação conforme os três critérios já anunciados: caracterização da agenda de pesquisa (quadro 1), modelo teórico (quadro 2) e modelo metodológico.

\section{Resultados}

O quadro 3 esquadrinha os objetos de investigação dos estudos em políticas locais de governo em 7 cenários que permitem melhor compreensão das questões relevantes da área. Como pode ser observado, as questões legítimas em termos de instituições estão associadas à análise daquilo que o poder executivo faz $(95,7 \%)$. Isso demonstra que há atenção especial às políticas dos governos. Considerando que o ambiente legislativo produz políticas de mais longo prazo, a ausência de estudos nos impede de compreendermos quais os motivos que dificultam que as políticas de esporte sigam esse caminho. Sua análise ajuda a tornar compreensível o estágio de desenvolvimento das políticas.

Especialmente no Brasil e na área da saúde, sobretudo, o judiciário tem sido acionado para equacionar problemas que surgem entre o direito do cidadão e a inação dos governos nesta matéria. A não institucionalização normativa do esporte no nível municipal no Brasil bloqueia essa agenda. No entanto, é uma janela de oportunidade importante para compreendermos a implementação de políticas esportivas em Portugal diante do conjunto de normas que imputam responsabilidades objetivas às câmaras municipais nesta matéria.

Outra característica importante, e com efeito na forma como pensamos a agenda, é que os analistas voltam seu olhar para o conjunto das ações do governo $(68,6 \%)$ e não para uma política específica. Excetuando as análises sobre o financiamento, a área da policy analysis não dispõe de instrumental teórico para esse nível de análise (a compreensão genérica das políticas). Desta forma, os achados têm baixa contribuição à formação da agenda de pesquisa.

Quanto à manifestação esportiva, a política de esporte tem sido privilegiada em detrimento da distinção entre rendimento (elite) e participação (para todos). Por outro lado, reconhecendo que há distinções importantes entre essas duas agendas, perdemos uma compreensão mais refinada do fenômeno.

Considerando que o instrumental teórico é pouco afeito aos parâmetros da policy analysis, essa foi a classificação mais difícil e que tornou precário os parâmetros sujeitos a erros de interpretação. Apesar de ser apenas uma simplificação esquemática para tornar mais 
Santos, E. S., \& Carvalho, M. J. (2020). Produção de pesquisa em políticas públicas locais de desporto no Brasil e em Portugal: agenda em formação

compreensível a complexidade da produção da política pública, as fases permitem uma observação mais acurada sobre como a política é produzida, implementada e avaliada.

Foi possível identificar que o trabalho de Werle (2010) anuncia a preocupação de compreender como se dá a tomada de decisão na Secretaria Municipal de Esporte de Florianópolis e o papel da participação popular. No entanto, o instrumental teórico não permitiu avançar na produção de resultados nos moldes propostos pela policy analysis. Também de forma precária, pelos mesmos motivos anunciados anteriormente, encontramos sete trabalhos que tentam precariamente compreender a implementação e trinta e três, a avaliação. Para 41,4\% dos trabalhos, o material empírico tornou a classificação pouco segura.

A fase da agenda/formulação, que possui diferentes modelos teóricos à análise, ainda não chamou atenção dos pesquisadores dos dois países. Mesmo diante da precariedade da estratégia utilizada para identificação das fases $\left[1^{\circ}\right.$. título; $2^{\circ}$. palavra-chave; $3^{\circ}$. resumo (justificativa ou objetivo)], encontramos as palavras "implementação" e "avaliação" em apenas 12,9\% (9) dos trabalhos. Ou seja, no mínimo essa designação não é utilizada para estruturar os trabalhos em políticas esportivas locais no Brasil e em Portugal.

Se por um lado o modelo de descentralização revela que os municípios possuem sua própria agenda na área esportiva [bottom-up $(82,9 \%)$, a descentralização top-down, no caso do Brasil, aponta para a importância que o Governo Federal assumiu, após 2003, na agenda esportiva nacional com a implementação dos programas Esporte e Lazer da Cidade (PELC) e Segundo Tempo (PST).

Como pode ser observado, há prevalência de estudos de caso $(45,7 \%)$. A atenção dos pesquisadores tem se voltado para o que acontece na política municipal de uma dada realidade. Apesar dos estudos comparativos formarem maioria (54,3\%), sua distribuição anuncia a riqueza do objeto de pesquisa, principalmente nos estudos sobre financiamento e implementação de programas top-down.

Apesar de existirem três trabalhos que tratam da produção legislativa, eles não discutem a dinâmica da produção das normas (das ideias e agendas e embates políticos em torno da votação) e nem o trabalho em torno das comissões, espaço de maior enfrentamento dos grupos de pressão.

Há uma cultura acadêmica em torno da policy analysis em políticas esportivas locais que impõe limitações importantes ao desenvolvimento do subcampo. A carência de estudos sobre o legislativo, a prevalência de análises gerais sobre os governos e não de políticas 
Santos, E. S., \& Carvalho, M. J. (2020). Produção de pesquisa em políticas públicas locais de desporto no Brasil e em Portugal: agenda em formação

específicas, a inexistência de estudos sobre agenda/formulação e das análises sobre o ambiente legislativo dificultam uma compreensão mais sofisticada do objeto de investigação.

Quadro 3 - Características da definição do objeto

\begin{tabular}{|c|c|c|c|}
\hline \multicolumn{4}{|c|}{ Instituição do Estado } \\
\hline Executivo & Legislativo & \multirow{2}{*}{\multicolumn{2}{|c|}{$\begin{array}{c}\text { Judiciário } \\
0 \%(0)\end{array}$}} \\
\hline $95,7 \%(67)$ & $4,3 \%(3)$ & & \\
\hline \multicolumn{4}{|c|}{ Política } \\
\hline $\begin{array}{l}\text { Específica } \\
31,4 \%(22)\end{array}$ & & \multicolumn{2}{|c|}{$\begin{array}{c}\text { Gestão } \\
68,6 \%(48)\end{array}$} \\
\hline \multicolumn{4}{|c|}{ Manifestação Esportiva } \\
\hline $\begin{array}{c}\text { Política esportiva } \\
87,1 \%(61)\end{array}$ & $\begin{array}{l}\text { Rendimento } \\
4,3 \%(3)\end{array}$ & \multicolumn{2}{|c|}{$\begin{array}{l}\text { Participação } \\
8,6 \% \text { (6) }\end{array}$} \\
\hline \multicolumn{4}{|c|}{ Fases da política } \\
\hline Agenda/formulação & Implementação & \multirow{2}{*}{\multicolumn{2}{|c|}{$\begin{array}{l}\text { Avaliação } \\
47,1 \% \text { (33) }\end{array}$}} \\
\hline $1,4 \%(1)$ & $10 \%(7)$ & & \\
\hline \multicolumn{4}{|c|}{ Modelo de descentralização } \\
\hline $\begin{array}{l}\text { Top-down } \\
17,1 \%(12)\end{array}$ & & \multicolumn{2}{|c|}{$\begin{array}{l}\text { Bottom-up } \\
82,9 \%(58)\end{array}$} \\
\hline \multicolumn{4}{|c|}{ Dimensão geográfica } \\
\hline RMou & Estadual & Regional & Nacional \\
\hline $45,7 \%(32)$ & $22,9 \%(16)$ & $2,9 \%(2)$ & $10 \%(7)$ \\
\hline \multicolumn{4}{|c|}{ Ambiente Legislativo } \\
\hline $\begin{array}{c}\text { Produção das leis } \\
0 \%(0)\end{array}$ & & $\begin{array}{c}\text { Comis } \\
0 \% \\
\end{array}$ & \\
\hline
\end{tabular}

Fonte: Dados da pesquisa.

O debate em torno dos modelos teóricos é um continuum que distancia os trabalhos do âmbito da policy analysis. A dificuldade em caracterizar a fase do trabalho impõe restrições na utilização dos modelos criados especialmente para a implementação e avaliação.

São poucos os trabalhos que utilizam modelos teóricos usados na policy analysis. Mesmo não sendo um modelo pouco usual no âmbito da policy analysis, o trabalho "Em tempos de megaeventos: as políticas públicas de esportes em uma cidade do interior de Minas Gerais" construiu sua ferramenta metodológica com base no materialismo histórico (MACIEL et al, 2014).

Outros trabalhos, que buscam associar os impactos do federalismo e da descentralização à implementação das políticas, se aproximam do neo-institucionalismo. Nesse conjunto de trabalhos, a política pública, especialmente seu financiamento, aparecem como variável dependente e os partidos (Santos, 2012), as instituições de governo (Santos \& Starepravo, 2018a; Santos, 2013) ou a capacidade fiscal (Santos \& Starepravo, 2018b) aparecem como 
Santos, E. S., \& Carvalho, M. J. (2020). Produção de pesquisa em políticas públicas locais de desporto no Brasil e em Portugal: agenda em formação

elementos explicativos (instituições) do investimento na Função Desporto e Lazer (FDL). No entanto, para além dos achados científicos desses trabalhos, eles carecem de instrumental metodológico mais sofisticado para produzirem evidências robustas.

Ou seja, os trabalhos confirmam as preocupações de diferentes autores que têm analisado a produção acadêmica brasileira em torno das políticas públicas de esporte que denunciam a falta de modelos teóricos à análise das políticas públicas (Starepravo, Afonso \& Ferreira, 2008; Starepravo, Nunes \& Marchi Jr., 2009; Godoy \& Vendruscolo, 2011; Starepravo, Souza \& Marchi Jr, 2011).

No entanto, alguns estudos têm sido produzidos utilizando de forma qualificada os modelos teóricos oriundos da ciência política. Bueno (2008) utilizou a teoria da Advocacy Coalition Framework (ACF) para analisar o predomínio do esporte rendimento na política esportiva brasileira. A análise de redes foi utilizada por Lorenzo (2008) para avaliar a experiência de participação no Conselho Nacional do Esporte. Mendes (2017) utilizou o mesmo referencial para analisar a participação da Frente Parlamentar do Esporte e da Comissão do Esporte da Câmara na construção das políticas públicas de esporte no Brasil. Carvalho (2013) utilizou ideias, atores e instituições para analisar a formulação de políticas de esporte no Brasil. Carvalho (2014) demonstrou como a cooperação descentralizada realizada pela experiência espanhola para implementação da Olimpíada de 1992 pode servir como parâmetro para a experiência brasileira, Olimpíada do Rio de Janeiro. Vieira (2010) utilizou conceitos teóricos associados à avaliação de projetos, programas e políticas públicas para avaliar o Programa Esporte e Lazer da Cidade (PELC). Santos (2019) realizou trabalho utilizando o modelo de ciclos políticos eleitorais para analisar com dados em painel o desempenho dos municípios da região Sul do Brasil no financiamento das políticas públicas de esporte.

Apesar de não anunciar de forma clara o modelo teórico associado ao Novo Gerencialismo Público (New Public Management), Sousa et al (2010) se utilizam de todo ferramental analítico vinculado a esse referencial para apresentar um modelo de avaliação e monitoramento do PELC e do PST.

Como podemos perceber, todos esses trabalhos demonstram que no Brasil há uma preocupação em se utilizar modelos teóricos oriundos do campo da policy analysis, mesmo que em sua maioria produzidos fora da área da educação física. Importante salientar que são trabalhos construídos entre o final dos anos 2000 e a década seguinte, demonstrando a juventude da iniciativa. 
É sintomático que $100 \%$ dos trabalhos sejam descritivos. Mesmo aqueles trabalhos que identificam variáveis independentes importantes à melhor compreensão da política (Santos, 2012; Santos \& Starepravo, 2018; Santos, 2013; Santos \& Starepravo, 2018) não se utilizam de instrumental analítico que permite produzir evidências robustas.

São trabalhos que, sob o olhar da policy analysis, têm sua contribuição limitada, segundo Frey (2000) e Arretche (2003), o que dificulta a estruturação de um campo de investigação sólido do ponto de vista teórico e metodológico. Isto porque, como revela Souza (2003, p. 16), "as disciplinas que contam com uma certa unidade de métodos e com contornos mais nítidos em relação ao seu objeto têm mais possibilidades de gerar conhecimento cumulativo".

O uso de narrativas pouco pautadas por modelos teóricos e metodológicos da policy analysis produz fragilidades analíticas (Souza, 2003). No âmbito dos trabalhos sobre as políticas de esporte no Brasil, Castro et al (2012) já haviam alertado para a falta de clareza teórica e metodológica. São trabalhos que apresentam hipóteses plausíveis sobre as políticas, mas que não anunciam ou testam as hipóteses causais (Arretche, 2003).

Há claramente, diante dos dados aqui analisados, ausência de uma agenda de pesquisa no Brasil e em Portugal, nos termos colocados por Melo (1999). São coleções de fatos, embora alguns trabalhos apresentem riqueza de dados, mas que não são suficientes para o desenvolvimento do campo (Arretche, 2003).

As questões legítimas não podem ser pensadas apartadas das implicações teóricas e metodológicas da escolha. Para 50\% (35) dos trabalhos que retratam as políticas de gestão, excetuando as análises de financiamento de descentralizações top-down, parte do princípio de que todas as políticas dos governos tiveram motivações comuns e/ou mesmos atores participando na definição de todas as políticas. O mesmo raciocínio pode ser estendido a outra característica dos trabalhos, política esportiva (Manifestação esportiva). Todos são trabalhos que não apontam uma política específica.

A existência de uma política específica é o ponto de partida para que possamos utilizar a maior parte do ferramental descrito no quadro 2. Portanto, a escolha do objeto de investigação define, em boa medida, o quadro teórico e metodológico. Sob essa unidade, não é possível dizer que os trabalhos apresentam uma agenda de pesquisa nos termos colocados pela policy analysis. Como destaca Kuhn (1976), a escolha dos fatos a serem analisados necessita de um corpo de 
Santos, E. S., \& Carvalho, M. J. (2020). Produção de pesquisa em políticas públicas locais de desporto no Brasil e em Portugal: agenda em formação

crenças teóricas e metodológicas que orientem sua seleção para desenvolverem um campo disciplinas.

O que existe no âmbito da análise de políticas públicas desportivas locais no Brasil e em Portugal é a proliferação de análises descritivas que não são suficientes à constituição de uma agenda de pesquisa na esfera da policy analysis.

\section{Conclusão}

Diferentes trabalhos produzidos no Brasil sobre a produção acadêmica em torno das políticas públicas indicam a existência de uma agenda de pesquisa e da necessidade de se superar alguns problemas causados pela fragilidade teórica dos trabalhos. O presente trabalho buscou analisar esse mesmo quadro a partir de dois ingredientes adicionais: os trabalhos que tratam das políticas públicas locais de esporte e aqueles que retratam as experiências do Brasil e de Portugal.

O levantamento bibliográfico permitiu encontrar 70 artigos científicos publicados, 62 do Brasil e oito de Portugal. Esse resultado evidencia que o tema tem chamado atenção de pesquisadores dos dois países. Ou seja, a esfera local tem estado no horizonte de preocupações das comunidades científicas. Considerando o número de agências implementadoras de políticas [Brasil (5.570) e Portugal (308)], não é possível conhecer a política nacional dos países sem trazer à superfície do debate o comportamento da esfera local de poder. $\mathrm{O}$ volume de trabalho (70) e o número de pesquisadores (126) envolvidos indicam que há um esforço importante em melhor compreender essa realidade. A questão é saber se esse empreendimento está sendo construído com bases sólidas, a partir de uma agenda de pesquisa no âmbito da policy analysis.

As evidências aqui encontradas nas temáticas de estudo, nos modelos teóricos e metodológicos utilizados indicam que estamos longe de construir uma agenda de pesquisa capaz de romper os limites impostos, especialmente, pelas análises descritivas. Elas não são suficientes para identificar o peso das variáveis intervenientes na definição das políticas, independentemente de qual etapa do ciclo que se refere a análise.

A análise de políticas públicas desportivas locais no Brasil e em Portugal ainda está em uma fase inicial dos estudos em que a preocupação de descrever o fenômeno da participação da esfera local de poder na definição das políticas parece ser mais importante do que revelar os fatores que afetam o desenvolvimento das políticas. A utilização de modelos teóricos e 
Santos, E. S., \& Carvalho, M. J. (2020). Produção de pesquisa em políticas públicas locais de desporto no Brasil e em Portugal: agenda em formação

metodológicos já consolidados, principalmente da ciência política, irá contribuir de maneira decisiva à superação do atual estágio de desenvolvimento dos estudos.

\section{Referências}

Almeida, B. S., \& Mezzadri, F. M. (2017). Quadros conceituais em políticas públicas: aplicações e possibilidades para a área do desporto. Caderno de Educação Física e Desporto, 15(1), 41-52.

Amaral, S. C. F., \& Pereira, A. P. C. (2009). Reflexões sobre a produção em políticas públicas de educação física, esporte e lazer. Revista brasileira de ciências do esporte, 31(1), 41-56.

Amaral, S. C. F., Ribeiro, O. C. F., \& Silva, D. S. (2014). Produção científico-acadêmica em Políticas Públicas de Esporte e Lazer no Brasil. Motrivivência, 26(42), 27-40.

Arretche, M. Dossiê agenda de pesquisa em políticas públicas. Revista Brasileira de Ciências Sociai, 18 (51), 7-10.

Bueno, L. (2008). Políticas Públicas do Esporte no Brasil: razões para o alto rendimento. (Tese de Doutorado). Escola de Administração de Empresas de São Paulo, São Paulo, Brasil.

Capella, A. C. N. (2006). Perspectivas Te ricas sobre o Processo de Formula o de políticas públicas. $B I B, 61$.

Capes. Portal.periódicos.capes. Disponível em: 〈http://www.periodicos. capes.gov.br>. Acesso em: 18 nov. 2019.

Carvalho, C. M. (2013). Esporte como política pública: um estudo sobre o processo de formulação da política de esporte no Brasil. (Dissertação de Mestrado), Universidade Federal de São Carlos, São Carlos, Brasil.

Carvalho, J. M. (2014). Os jogos olímpicos, a cooperação descentralizada e a aplicação de políticas públicas: o modelo de Barcelona 92 para o Rio de Janeiro 2016. Revista de Estudos Universitários, 40, 259-274.

Castro, S.B.E. de et al., (2012). O estado da arte em políticas sociais de esporte e lazer no Brasil (2000-2009). Pensar a Prática, 15(2), 272-550.

Donaldson, T. \& Preston, L.E. (1995). The stakeholder theory of the corporation: concepts, evidence, and implications. The Academy of Management Review, 20(1), 65-91.

Frey, K. (2000). Políticas públicas: um debate conceitual e reflexões referentes à prática da análise de políticas públicas no Brasil. Planejamento e Políticas Públicas, (21), 211-259.

Giustini D, B. A (2005). Look at Google Scholar, PubMed, and Scirus: comparisons and recommendations. Journal of the Canadian Health Libraries Association, 26(3), 85-9. 
Starepravo, F. A., Afonso, G., \& Ferreira, A. L. P. Produção do GTT de políticas públicas no XV Congresso Brasileiro de Ciências do Esporte: possibilidades teóricas de análise das políticas públicas de esporte e lazer. In Anais $1^{\circ}$ Encontro da ALESDE (pp 1-8). Curitiba.

Godoy, L. \& Vendruscolo, R. (2011). Desafios da produção de conhecimentos sobre políticas públicas para o esporte e o lazer no Brasil. In Anais Congresso Brasileiro de Sociologia. Curitiba.

Goodin, R. E., Rein, M., \& Moran, M. (2009). Overview of Public Policy: the public and its policies. In R. E. Goodin (Ed.), The Oxford Handbook or Political 235 Science (pp. 885918). Oxford University Press.

Houlihan, B. (2005). Public sector sport policy: developing a framework for analysis. International Review for the Sociology of Sport, 40(2), 163-185.

Howlett, M., Ramesh, M. \& Perl, A. (2009). Studying public: policy cycles and policy subsystems. Oxford University Press. Ontario.

Kocian, R. C., \& da Silva, C. L. (2016). Políticas públicas e os equipamentos esportivos de lazer: produção acadêmica de 2008 a 2014. Licere, 19(3), 185-206.

Kuhn, Thomas. (1976), A estrutura das revoluções científicas. São Paulo, Perspectiva.

Lorenzo, M. C. (2008) Análise das práticas de participação em conselhos deliberativos de política: o caso do Conselho Nacional do Esporte. (Dissertação de Mestrado), Universidade de Brasília, Brasília, Brasil.

Maciel, T. B., dos Reis, H. K., de Melo Rail, R., \& de Oliveira Correa, P. H. (2014). Em tempos de megaeventos: as políticas públicas de esportes em uma cidade do interior de Minas Gerais. Motrivivência, 26(42), 72-85.

Melo, M. A. (1999). Estado, governo e políticas públicas. In: Miceli, S. (Org.). O que ler na ciência social brasileira. São Paulo/Sumaré: Anpocs/Capes, pp. 59-99.

Mendes, A. (2013). Política pública de esporte: considerações sobre gestão, instituições políticas e método de pesquisa no brasil. Revista Intercontinental de Gestão Desportiva, 3. $1-19$.

Rijo, V. A. (2018). Investigação científica em gestão do desporto: análise das dissertações de mestrado em Direção e Gestão Desportiva a partir de três universidades públicas nacionais (Master's thesis), Universidade de Évora, Évora, Portugal.

Rojo, J. R., Mezzadri, F. M., \& Moraes, M. (2019). A Produção do Conhecimento sobre Políticas Públicas para o Esporte e Lazer no Brasil: Uma Análise dos Pesquisadores e Instituições. PODIUM Sport, Leisure and Tourism Review, 8(1), 128-139.

Sampaio, R. F., \& Mancini, M. C. (2007). Systematic review studies: a guide for careful synthesis of the scientific evidence. Brazilian Journal of Physical Therapy, 11(1), 83-89. 
Santos, E. S., \& Carvalho, M. J. (2020). Produção de pesquisa em políticas públicas locais de desporto no Brasil e em Portugal: agenda em formação

Santos, A. L. F., Batista, M. C. A. \& Araújo, A. V. (2007). A produção do conhecimento em política pública para educação física, esporte e lazer: o GTT 10 em Pernambuco. In Anais do XV Congresso Brasileiro de Ciências do Esporte (pp 1-10). Recife.

Santos, E. S. (2013). Ambiente Institucional e difusão do Programa Segundo Tempo: uma análise do desempenho de 2005 a 2010. Pensar a Prática, 16(1), 35-53.

Santos, E. S. (2012). As coalizões e os partidos políticos na difusão do Programa Segundo Tempo. Licere, 15(3), 1-26.

Santos, E. S., \& Starepravo, F. A. (2018). Análise da capacidade fiscal per capita na descentralização do Programa Segundo Tempo/ME junto aos municípios. Movimento, 24(4), 1097-1110.

Santos, E. S., \& Starepravo, F. A. (2018). Estrutura político-administrativa dos governos municipais do Piauí e investimento no esporte e no lazer. Licere, 21(4), 166-183

Secchi. L. (2022). Políticas Públicas: Conceitos, Esquemas de Análise, Casos Práticos. Cengage Learning. 2011.

Shultz, M. (2007). Comparing test searches in PubMed and Google Scholar. Journal of the Medical Library Association, 95(4), 442.

Sousa, D. P., Gabriel, B. J., Antunes, A. C., Pedroso, B., Oliveira Junior, C. R., \& de Freitas Jr, M. A. (2016). As produções do periódico "Pensar a Prática" que relacionaram os seus objetos às políticas públicas de esporte e lazer (1998-2015). Pensar a Prática, 19(3), 612626

Sousa, E. S. D., Noronha, V., Ribeiro, C. A., Teixeira, D. M. D., Fernandes, D. M., \& Venâncio, M. A. D. (2010). Sistema de monitoramento \& avaliação dos programas Esporte e Lazer da Cidade e Segundo Tempo do Ministério do Esporte.

Souza, C. (2003). " Estado do campo" da pesquisa em políticas públicas no Brasil. Revista Brasileira de Ciências Sociais, 18(51), 15-20.

Souza, C. (2006). Políticas públicas: uma revisão da literatura. Sociologias, 8 (16), 20-45.

Starepravo, F. A., Afonso, G., \& Ferreira, A. L. P. Produção do GTT de políticas públicas no XV Congresso Brasileiro de Ciências do Esporte: possibilidades teóricas de análise das políticas públicas de esporte e lazer. In Anais $1^{\circ}$ Encontro da ALESDE (pp 1-8). Curitiba.

Starepravo, F. A., de Souza, J., \& Junior, W. M. (2011). Políticas públicas de esporte e lazer no Brasil: uma proposta teórico-metodológica de análise. Movimento, 17(3), 233-251.

Starepravo, F. A., Nunes, R. J. S., \& Marchi Jr, W. (2009). Agenda de pesquisa em políticas públicas de esporte e lazer: uma leitura a partir do GTT de Políticas Públicas no XV Congresso Brasileiro de Ciências do Esporte. In Anais do XVI Congresso Brasileiro de 
Santos, E. S., \& Carvalho, M. J. (2020). Produção de pesquisa em políticas públicas locais de desporto no Brasil e em Portugal: agenda em formação

Ciências do Esporte [e] III Congresso Internacional de Ciências do Esporte (pp 1-14). Salvador. 